International Journal of Engineering \& Technology, $7(2.15)(2018) 154-157$
International Journal of Engineering \& Technology
SPC
Website: www.sciencepubco.com/index.php/IJET
Research Paper

\title{
An automated grading system for diabetic retinopathy using curvelet transform and hierarchical classification
}

\author{
Fanji Ari Mukti ${ }^{1}$, C. Eswaran ${ }^{1}$, Noramiza Hashim ${ }^{1}$, Ho Chiung Ching ${ }^{1}$, Mohamed Uvaze Ahamed Ayoobkhan ${ }^{2 *}$ \\ ${ }^{1}$ Centre for Visual Computing, Faculty of Computing and Informatics, Multimedia University, Cyberjaya, Malaysia \\ ${ }^{2}$ Department of Computer Science and Engineering, School of Engineering and Technology, Jain University, Bangalore, India \\ *Corresponding author E-mail: mohamed.sha33@gmail.com
}

\begin{abstract}
In this paper, an automated system for grading the severity level of Diabetic Retinopathy (DR) disease based on fundus images is presented. Features are extracted using fast discrete curvelet transform. These features are applied to hierarchical support vector machine (SVM) classifier to obtain four types of grading levels, namely, normal, mild, moderate and severe. These grading levels are determined based on the number of anomalies such as microaneurysms, hard exudates and haemorrhages that are present in the fundus image. The performance of the proposed system is evaluated using fundus images from the Messidor database. Experiment results show that the proposed system can achieve an accuracy rate of $86.23 \%$.
\end{abstract}

Keywords: Automated screening system; Curvelet transform; Diabetic retinopathy; Fundus image; SVM classifier.

\section{Introduction}

Diabetic Retinopathy (DR) is one of the complications of diabetes that affects the eyes. Diabetes Mellitus is one of the most common metabolic disorders around the world and is characterized by high blood sugar levels [1-2]. The first stage of DR is known as the Non-Proliferative DR (NPDR) and the second or the advanced stage is known as the Proliferative DR (PDR). If left untreated for a prolonged time, DR could lead to many complications resulting in total blindness. Hence, researchers are attempting to devise different screening methods for the detection of DR at an initial stage using the anomalies present in the fundus images and applying different sets of grading rules [3-4]. In this paper, an automated screening system for the grading of NPDR based on the fundus images is presented. The proposed method makes use of two anomalies, namely Microaneurysm (MA) and Haemorrhage (HA) to grade NPDR. MA is focal dilatation of retinal capillaries and indicates the leakage of blood from an artery or vein in the back of an eye.

It can occur due to causes such as high blood pressure or vascular diseases, but the most common cause is DR. MA appears as a tiny black spot on retinal fundus images as can be observed on Figure 1 .

As capillary's walls weaken, ruptures may occur and cause HA, the second anomaly of DR which is used for grading NPDR level. Figure 2 shows how HA appears in a fundus image.

The severity level of DR is classified into four grades namely normal, mild, moderate and severe based on the following rules which are formulated by the ophthalmology specialists involved in the Messidor program [11].

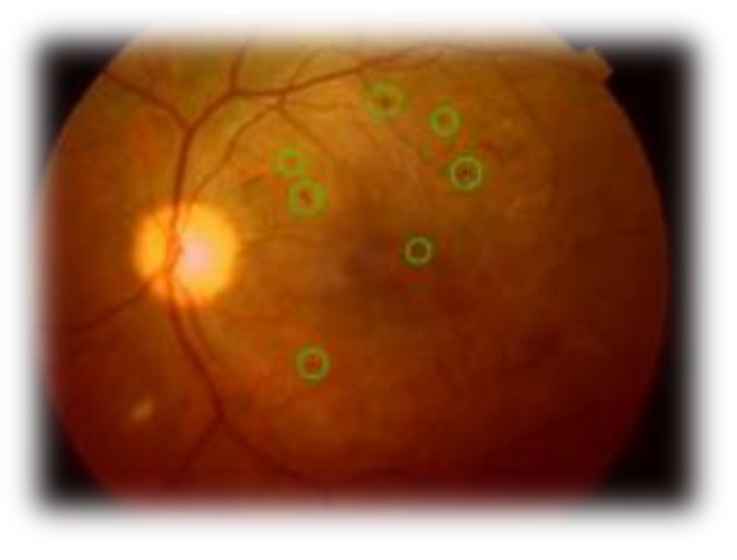

Fig. 1: Microaneurysms

1. If none of the two anomalies is present, the fundus image is classified as normal.

2. Mild Non-Proliferative Retinopathy (Mild NPDR) is characterized by the anomaly MA. A fundus image will be classified as Mild NPDR if it contains 0 to 5 MA but no HA. It is the lowest severity of DR.

3. Moderate Non-Proliferative Retinopathy (Moderate NPDR) contains 5 to $15 \mathrm{MA}$ or 0 to $5 \mathrm{HA}$.

4. Severe Non-Proliferative Retinopathy (Severe NPDR) is characterized by the presence of more number of anomalies MA and HA. There should be more that 15 MA or more than 5 HA. This severity stage is dangerous and should be referred to an Ophthalmologist without delay.

When a patient is diagnosed with DR, the best way to prevent blindness is to rate the severity of the DR and recommend the best follow up treatment according to the severity level. 


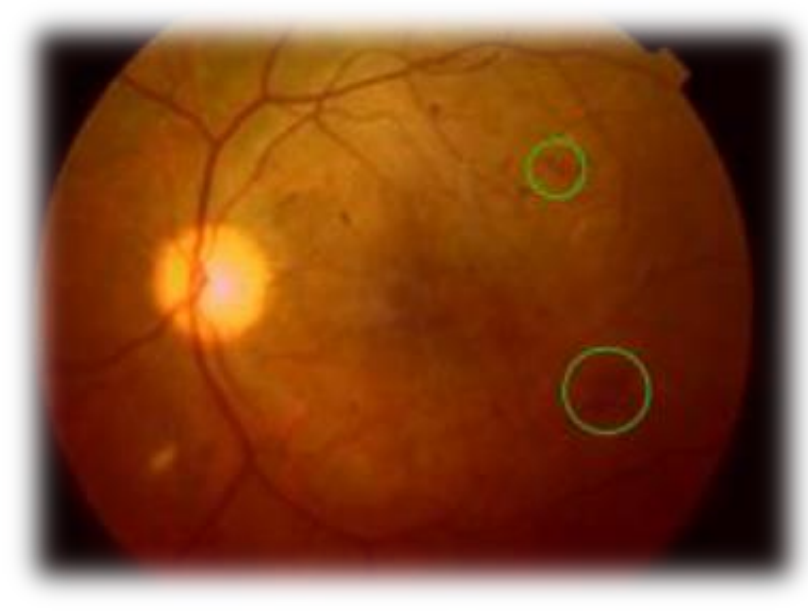

Fig. 2: Haemorrhages

In this paper, we proposed an automated DR severity grading system by applying the Fast Discrete Curvelet Transform features [5] and hierarchical classification using Support Vector Machine Classifier [6] (SVM).

\section{Related works}

In [2] have proposed an automatic triage scenario for automatic screening of Diabetic Retinopathy in the retinal fundus images. The authors have used the concept of visual dictionaries, where each image is treated as a collection of regions. The visual dictionaries constitute a robust representation approach where each image is treated as a collection of regions. The image is represented according to the visual words it contains. To extract the features for each region, the authors have made use of the Points of Interests (PoI) approach with Speeded-Up Robust Features (SURF) as the feature extractor. For the final classification, that is to detect the presence of anyone of the anomalies, Support Vector Machine (SVM) has been used.

In [7] have proposed a system to extract candidate regions for possible HE using filter banks. The proposed approach makes use of three stages: the first stage is the retinal image acquisition and pre-processing, second stage is the candidate exudate region detection and elimination of the optic disc and the last stage is the feature set formulation and classification of regions as exudate or non-exudate regions. A Gaussian Bayes decision rule has been used to identify exudate and non-exudate pixels using a feature set for each region.

In [8] have proposed a classification system to classify two severity levels namely moderate NPDR and severe NPDR. The proposed system has three stages: the first stage is exudate segmentation based on the morphology method, the features from the segmented image are extracted in the second stage and classified into different severity levels using soft margin SVM in the last stage.

In [9] have proposed another novel approach for detection and grading of macular edema to assist ophthalmologists in detecting the disease. An ensemble of Gaussian mixture model and SVM is presented as a new hybrid classifier for improved exudate detection even in the presence of other bright lesions. The proposed system is also divided into three stages: macula detection in which vascular pattern is detected and then macula feature is extracted to determine the coordinates, exudate detection where the optic disc is localized and then candidate exudates are extracted and finally macular edema grading where the images are classified into three classes: non clinically significant macular edema, clinically significant macular edema and normal.

In [10] have proposed an algorithm based on curvelet transform to separate red lesions from the rest of the colour retinal image. A new illumination equalization algorithm has been introduced to prevent fovea from being considered as red lesion. The illumina- tion equalization algorithm is applied to the green plane of retinal image. Digital curvelet transform has been applied to produce enhanced image and appropriate threshold has then been applied to separate the lesions.

\section{Fast discrete curvelet transform}

In the proposed method, Fast Discrete Curvelet Transform (FDCT) which was introduced by [5] has been utilized to extract the features. There are two implementations of FDCT; the first one is based on unequally spaced fast Fourier transforms while the second one is based on the wrapping of specially selected Fourier samples [4]. These two digital transformations differ in the choice of the spatial grid used to translate curvelets at each scale and angle. FDCT is implemented by first taking 2D Fast Fourier transform of the input fundus image. The frequency space is divided into dyadic annuli based on the concentric squares and each annulus is then further subdivided into trapezoidal regions as shown in Figure 3. Each of the wedges of the trapezoidal regions is smooth, nonnegative and real-valued. Curvelet coefficients are the inverseFast Fourier transform of each smoothed wedge.

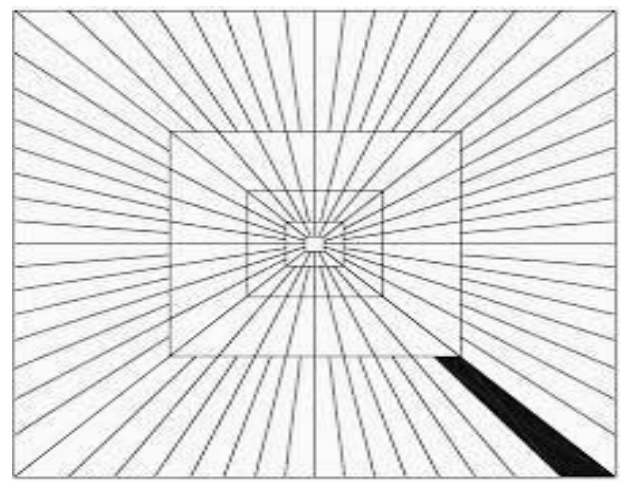

Fig. 3: Basic digital tiling for trapezoidal region

\section{Methodology}

Figure 4 shows the block diagram illustrating the steps involved in the proposed method.

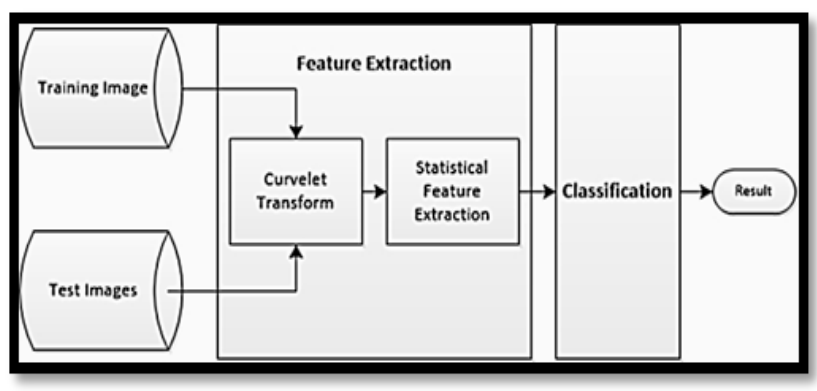

Fig. 4: Block diagram of the proposed method

Wrapping-based fast discrete curvelet transform is made use of for extracting the features. Several sets of digital curvelet coefficients indexed by a scale parameter, an orientation parameter and a spatial location parameter are obtained. From the curvelet coefficients, the following statistical features are extracted:

1. Entropy

2. Mean

3. Standard Deviation

4. Maximum Probability

5. Homogeneity

The statistical features from the curvelet coefficients are used to represent the whole retinal fundus image in multiscale decomposi- 
tion level. Several experiments are conducted by varying the number of scales and angles in order to determine, which combination of scale and angle yields the highest accuracy.

The statistical features obtained from FDCT coefficients are applied to a support vector machine (SVM) classifier [3]. SVM classifier is a binary classifier which means that the result of the classifier will either be positive or negative. The binary nature of the SVM classifier is further expanded by the use of Hierarchical classification to create a multi-level classification.

In the hierarchical classification, the input images are first classified into two classes, namely, normal and abnormal. An image is considered to be normal if it does not contain any of the anomalies. On the other hand, if it contains any one of the anomalies, it is considered abnormal. In the next stage, an image which is classified as abnormal is further classified into either Mild NPDR or others based on the pattern of Curvelet coefficients. The SVM is trained to recognize the pattern of Mild NPDR during the training phase [3]. After separating the Mild NPDR fundus images, the system will then finally classify the remaining images into Moderate NPDR or Severe NPDR. Figure 5 illustrates the hierarchical classification system.

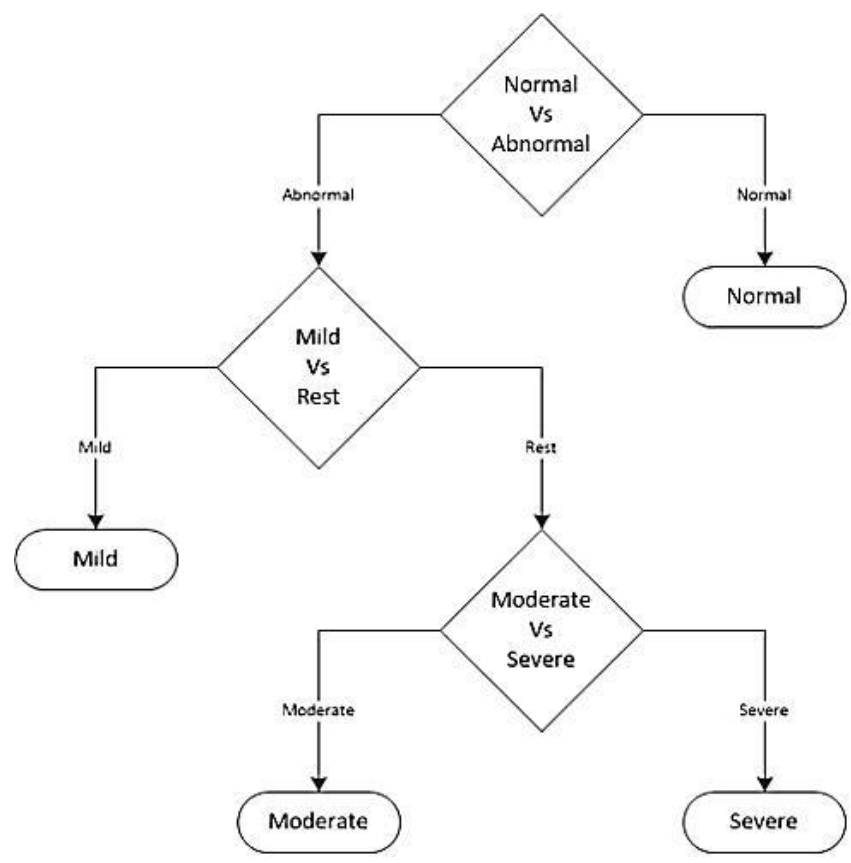

Fig. 5: Hierarchical classification

Experiments are conducted using the well-known Messidor database provided by the Messidor program partners. The Messidor database was created to facilitate studies on computer-assisted diagnosis of DR. The database contains color images of the retina that were acquired using a retinograph. The examinations on those fundus images were performed by four ophthalmology departments involved in the Messidor program [11].

The Messidor database comprises two main database sets namely training set and evaluation sets. The training set consists of around 300 images. MA, HE, HA and NV are all marked individually on these images. The evaluation set consists of around 1,000 images. This set is especially for evaluating the performance of developed algorithms. The images in the evaluation set are annotated based on the severity level of DR. There are 450 normal images, 150 images with Mild NPDR, 150 images with Moderate NPDR and 150 images with Severe NPDR.

The four severity levels namely Normal, Mild NPDR, Moderate NPDR, Severe NPDR are represented as $0,1,2$ and 3 respectively. The determination of the severity levels is based on the rules described in section 1.

Experiments are performed using MATLAB software with image processing toolbox on a computer with the specifications: Intel i7
3630QM 2.4GHz, 8GB RAM, GeForce G650M 4GB graphics card.

\section{Results and discussion}

The performance of the proposed method is evaluated by conducting several experiments. The curvelet features are extracted using different scale and orientation parameter values. The chosen values for the scale parameter are 2, 3, 4, 5, 6, 7 and 8. Three orientation values namely 8,12 add 16 are used with each of the scale parameter. Table 1, 2 and 3 show some sample results obtained using the proposed method.

Table 1: Scale $=2$, Angle $=8$

\begin{tabular}{|c|c|c|c|c|c|}
\hline \multicolumn{6}{|c|}{ Scale $=2$, Angle $=8$} \\
\hline \multirow{7}{*}{ 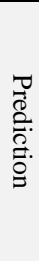 } & & Actu & Class & & \\
\hline & Class & $\begin{array}{l}\text { No } \\
\text { DR }\end{array}$ & $\begin{array}{c}\text { Mild } \\
\text { NPDR }\end{array}$ & $\begin{array}{c}\text { Moderate } \\
\text { NPDR }\end{array}$ & $\begin{array}{l}\text { Severe } \\
\text { NPDR }\end{array}$ \\
\hline & No DR & 300 & 14 & 9 & 14 \\
\hline & Mild NPDR & 37 & 127 & 6 & 11 \\
\hline & Moderate NPDR & 43 & 4 & 125 & 11 \\
\hline & Severe NPDR & 20 & 5 & 10 & 114 \\
\hline & Total Image in Class & 400 & 150 & 150 & 150 \\
\hline
\end{tabular}

Table 2: Scale $=4$, Angle $=12$

\begin{tabular}{|c|c|c|c|c|c|}
\hline \multicolumn{6}{|c|}{ Scale $=4$, Angle $=12$} \\
\hline \multirow{7}{*}{ 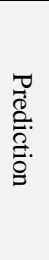 } & & Actu & Class & & \\
\hline & Class & $\begin{array}{l}\text { No } \\
\text { DR }\end{array}$ & $\begin{array}{c}\text { Mild } \\
\text { NPDR }\end{array}$ & $\begin{array}{l}\text { Moderate } \\
\text { NPDR }\end{array}$ & $\begin{array}{l}\text { Severe } \\
\text { NPDR }\end{array}$ \\
\hline & No DR & 309 & 7 & 9 & 14 \\
\hline & Mild NPDR & 37 & 125 & 9 & 11 \\
\hline & Moderate NPDR & 33 & 10 & 119 & 11 \\
\hline & Severe NPDR & 21 & 8 & 13 & 114 \\
\hline & Total Image in Class & 400 & 150 & 150 & 150 \\
\hline
\end{tabular}

Table 3: Scale $=7$, Angle $=16$

\begin{tabular}{c|c|c|c|c|c}
\hline \multicolumn{5}{c}{ Scale $=7$, Angle $=16$} \\
\hline & \multicolumn{5}{c}{ Actual Class } \\
\cline { 2 - 6 } & Class & $\begin{array}{c}\text { No } \\
\text { DR }\end{array}$ & $\begin{array}{c}\text { Mild } \\
\text { NPDR }\end{array}$ & $\begin{array}{c}\text { Moderate } \\
\text { NPDR }\end{array}$ & $\begin{array}{c}\text { Severe } \\
\text { NPDR }\end{array}$ \\
\hline & $\begin{array}{c}\text { No DR } \\
\text { Mild }\end{array}$ & 393 & 4 & 0 & 2 \\
\hline \multirow{8}{*}{} & $\begin{array}{c}\text { NPDR } \\
\text { Moderate } \\
\text { NPDR } \\
\text { Severe }\end{array}$ & 1 & 119 & 24 & 15 \\
& $\begin{array}{c}\text { NPDR } \\
\text { Total } \\
\text { Image in } \\
\text { Class }\end{array}$ & 1 & 6 & 97 & 9 \\
\hline
\end{tabular}

The total number of images used is 850 with 400 in the normal class and 150 in each of the other three classes (Mild, Moderate and Severe). The percentage of the correctly classified images gives the accuracy of the system. The diagonal entries in the table give the number of correctly classified images in each class. For example, in Table 1, the number of correctly classified images in Normal, Mild NPDR, Moderate NPDR and Severe NPDR classes are $300,127,125$ and 114 respectively. In other words, out of 850 images, 666 images are correctly classified yielding an accuracy value of 0.7835 or $78.35 \%$. The accuracy values obtained with different scale and orientation parameter values are shown in Figure 6. 


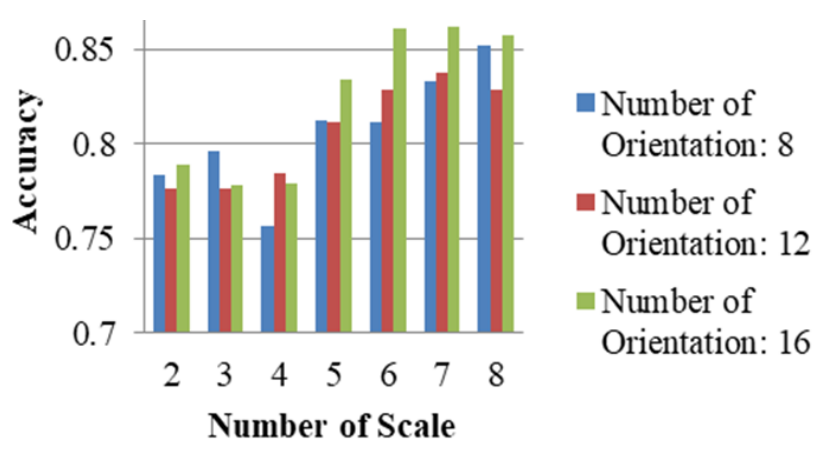

Fig. 6: Accuracy graph

As we can observe from Figure 6, the accuracy value increases as the number of scale increases and reaches a breaking point when the scale parameter value is 7 . The highest accuracy is obtained for scale and orientation parameter values 7 and 16 respectively. In this case, the proposed system yields an accuracy of $86.23 \%$.

\section{Conclusion}

An automatic grading system for DR using FDCT features with SVM Classifier has been presented in this paper. The grading of the images, the rules formulated by the specialists involved in the Messidor program has been employed. This system will be very useful for automatic screening for diabetic retinopathy disease. The proposed system will save time and effort and in addition, it will also avoid the need for experts. The proposed system has been tested using images from Messidor databases. It has been found from experimental results that this system yields an accuracy of $86.23 \%$.

\section{Acknowledgement}

The authors thank the Ministry of Higher Education (MOHE), Malaysia and Multimedia University for supporting this research through ERGS and PRGS Projects.

\section{References}

[1] Silink GRM, Tuomilehto J, Mbanya JC, Venkat Narayan KM \& Judy F (2010), Research priorities: Prevention and control of diabetes with a focus on low and middle income countries. World Health Organization.

[2] Rocha A, Carvalho T, Jelinek HF, Goldenstein S \& Wainer J (2012), Points of interest and visual dictionaries for automatic retinal lesion detection. IEEE Transactions on Biomedical Engineering 59, 2244-2253.

[3] Eswaran C, Mukti FA \& Hashim N (2014), Comparison of classifiers for retinal pathology images using SURF and Bag-ofWords Model. Proceedings of the International Conference on Artificial Intelligence and Pattern Recognition, pp. 72-78.

[4] Nor FN, Ang EL, Bavaanandan NG, Loong AM, Mohamad AS, Maziah I, Mohd. AMY, Nor AI, Nik MKNS, Roslin AAA \& Shelina OM (2011), Clinical practice guidelines-Screening of diabetic retinopathy. http://www.moh.gov.my/penerbitan/CPG2017/6601.pdf.

[5] Candes E, Demanet L, Donoho D \& Ying L (2006), Fast discrete curvelet transforms. Multiscale Modeling and Simulation 26, 861899.

[6] Burges CJ (1998), A tutorial on support vector machines for pattern recognition. Data Mining and Knowledge Discovery 2, 121-167.

[7] Akram MU, Tariq A, Anjum MA \& Javed MY (2012), Automated detection of exudates in colored retinal images for diagnosis of diabetic retinopathy. Applied Optics 51, 4858-4866.

[8] Tjandrasa H, Putra RE, Wijaya AY \& Arieshanti I (2013), Classification of non-proliferative diabetic retinopathy based on hard exudates using soft margin SVM. Proceedings of the IEEE International Conference on Control System, Computing and Engineering, pp. 376-380.
[9] Akram MU, Tariq A, Khan SA \& Javed MY (2014), Automated detection of exudates and macula for grading of diabetic macular edema. Computer Methods and Programs in Biomedicine 114, 141152.

[10] Esmaeili M, Rabbani H, Dehnavi AM \& Dehghani A (2010), A new curvelet transform based method for extraction of red lesions in digital color retinal images. Proceedings of the 17th IEEE International Conference on Image Processing, pp. 4093-4096.

[11] ADCIS SA (2017), Methods to evaluate segmentation and indexing techniques in the field of retinal ophthalmology (MESSIDOR). http://www.adcis.net/en/Download-Third-Party/Messidor.html. 